\title{
Sur certaines séries entières particulières
}

\author{
par
}

\section{Hubert Delange (Orsay)}

1. Introduction. Dans un exposé à la Conférence Internationale de Théorie des Nombres organisée à Zakopane (Pologne) en juillet 1997, en l'honneur du professeur Schinzel, l'auteur a démontré le résultat suivant : $\mu$ étant la fonction de Möbius, quand $x$ tend vers 1 par valeurs inférieures, alors que

$$
\sum_{n=1}^{\infty} \mu(n) x^{n}=o\left(\frac{1}{1-x}\right)
$$

on a

$$
\sum_{n=1}^{\infty} \mu(n) x^{n}=\Omega_{ \pm}\left(\frac{1}{\sqrt{1-x}}\right)
$$

(cette notation classique signifie que la limite supérieure du produit $\sqrt{1-x} \sum_{n=1}^{\infty} \mu(n) x^{n}$ est $>0$ et la limite inférieure est $\left.<0\right)$.

On va considérer ici, en même temps que la série ci-dessus, d'autres séries entières à coefficients réels ayant les mêmes propriétés, et liées aussi à des fonctions arithmétiques classiques.

Dans ce qui suit, les lettres $n$ et $k$ représentent des entiers $>0$ et la lettre $p$ est utilisée pour représenter les nombres premiers.

2. Nos résultats sont basés sur le théorème suivant :

THÉORÈME. Soit $a_{1}, a_{2}, \ldots$ une suite de nombres réels ayant les trois propriétés suivantes :

1) La série entière $\sum_{n=1}^{\infty} a_{n} x^{n}$ a pour rayon de convergence 1 ;

2) Quand $x$ tend vers $\infty, \sum_{n \leq x} a_{n}=o(x)$;

3) La série de Dirichlet $\sum_{n=1}^{\infty} a_{n} / n^{s}$ est convergente pour $\operatorname{Re} s>1$ et sa somme est une fonction de $s$ prolongeable par une fonction $F$ méromorphe dans un domaine $D$ contenant le demi-plan fermé $\operatorname{Re} s \geq 1 / 2$ ayant des pôles

1991 Mathematics Subject Classification: Primary 11A99. 
de partie réelle $1 / 2$, mais pas de pôle réel $\geq 1 / 2$ ni de pôle de partie réelle $\geq 1$ (ce qui est vrai en particulier si ses pôles dans le demi-plan $\operatorname{Re} s \geq 1 / 2$ sont les zéros de la fonction $\zeta)$.

Ces propriétés entraînent que, quand $x$ tend vers 1 ,

$$
\sum_{n=1}^{\infty} a_{n} x^{n}=o\left(\frac{1}{1-x}\right) \quad \text { et } \quad \sum_{n=1}^{\infty} a_{n} x^{n}=\Omega_{ \pm}\left(\frac{1}{\sqrt{1-x}}\right) .
$$

On voit facilement que la propriété 2 ) entraîne que, quand $x$ tend vers 1 ,

$$
\sum_{n=1}^{\infty} a_{n} x^{n}=o\left(\frac{1}{1-x}\right)
$$

(par exemple en remplaçant $a_{n}$ par $A_{n}-A_{n-1}$, où $A_{n}=\sum_{k=1}^{n} a_{k}$ pour $n \geq 1$ et $\left.A_{0}=0\right)$.

Notons que cette propriété a certainement lieu si la série $\sum_{n=1}^{\infty} a_{n} / n$ est convergente, car on sait que la convergence d'une série $\sum_{n=1}^{\infty} u_{n}$ entraîne que, quand $x$ tend vers $\infty, \sum_{n \leq x} n u_{n}=o(x)$.

Pour $0<x<1$, on peut poser $x=e^{-u}$, avec $u>0$. Quand $x$ tend vers 1, $u$ tend vers zéro et $1-x \sim u$.

Le résultat qui reste à démontrer est donc équivalent à

$$
\sum_{n=1}^{\infty} a_{n} e^{-n u}=\Omega_{ \pm}\left(u^{-1 / 2}\right) \quad \text { quand } u \text { tend vers } 0 .
$$

Notons que ce résultat entraîne que, quand $x$ tend vers $\infty$,

$$
\sum_{n \leq x} a_{n}=\Omega_{ \pm}\left(x^{1 / 2}\right) .
$$

En effet, en partant de ce que, pour $u>0$,

$$
\sum_{n=1}^{\infty} a_{n} e^{-n u}=u \int_{0}^{\infty} e^{-u x} A(x) d x, \quad \text { où } \quad A(x)=\sum_{n \leq x} a_{n},
$$

on voit facilement que, pour $\lambda>0$,

$$
\varlimsup_{u \rightarrow 0}\left(u^{\lambda} \sum_{n=1}^{\infty} a_{n} e^{-n u}\right) \leq \Gamma(\lambda+1) \varlimsup_{x \rightarrow \infty}\left(x^{-\lambda} A(x)\right)
$$

et

$$
\varliminf_{u \rightarrow 0}\left(u^{\lambda} \sum_{n=1}^{\infty} a_{n} e^{-n u}\right) \geq \Gamma(\lambda+1) \varliminf_{x \rightarrow \infty}\left(x^{-\lambda} A(x)\right) .
$$


3. La démonstration de (1) est basée sur le lemme suivant :

LEMME. $f$ étant une fonction réelle définie sur l'intervalle $[1, \infty[$, supposons que l'intégrale $\int_{1}^{\infty}\left(f(x) / x^{s+1}\right) d x$ a pour abscisse de convergence $\alpha>0$ et que, pour $\operatorname{Re} s>\alpha$,

$$
\int_{1}^{\infty} \frac{f(x)}{x^{s+1}} d x=F(s)
$$

où $F$ est une fonction méromorphe dans un domaine contenant le demi-plan fermé $\operatorname{Re} s \geq \alpha$.

Si $\alpha$ n'est pas un pôle de $F$, pour tout $\varepsilon \in] 0, \alpha[$ on a

$$
f(x)=\Omega_{ \pm}\left(x^{\alpha-\varepsilon}\right) \quad \text { quand } x \text { tend vers } \infty .
$$

Si $\alpha$ n'est pas un pôle de $F$ mais $F$ a des pôles de partie réelle $\alpha$, on a

$$
f(x)=\Omega_{ \pm}\left(x^{\alpha}\right) .
$$

Ce lemme résulte des lemmes IV 8 à IV 10, pages 97 et 98, de [1]. Ces lemmes sont déduits du lemme IV 7, qui les précède. Celui-ci est un théorème établi par Landau dans [3] (c'est l'analogue pour l'intégrale de Mellin de son célèbre théorème, établi aussi dans [3], d'après lequel, si une série de Dirichlet à coefficients $\geq 0$ a une abscisse de convergence $\sigma_{c}$ finie, $\sigma_{c}$ est un point singulier de la fonction qu'elle représente). Landau indique que c'est une amélioration d'un théorème de Phragmén.

4. Démonstration de (1). Soit $H(u)=\sum_{n=1}^{\infty} a_{n} e^{-n u}$ pour $u>0$. Nous devons démontrer que $H(u)=\Omega_{ \pm}\left(u^{-1 / 2}\right)$ quand $u$ tend vers zéro.

4.1. Notons d'abord que le produit $u e^{u / 2} H(u)$, qui est une fonction de $u$ continue sur l'intervalle ]0, $\infty$ [, tend vers zéro quand $u$ tend vers zéro et quand $u$ tend vers l'infini.

D'une part, quand $u$ tend vers zéro,

$$
H(u)=o\left(\frac{1}{1-e^{-u}}\right)=o\left(\frac{1}{u}\right)
$$

d'après ce qu'on a remarqué après avoir énoncé le théorème.

D'autre part, quand $u$ tend vers $\infty$, comme $e^{-u}$ tend vers zéro, $H(u) / e^{-u}$ tend vers $a_{1}$. Il existe donc $M>0$ tel que $\left|u e^{u / 2} H(u)\right| \leq M$, c'est-à-dire

$$
|H(u)| \leq M u^{-1} e^{-u / 2} \quad \text { pour tout } u>0 .
$$

4.2. Il résulte de là que l'intégrale $\int_{0}^{\infty} u^{s-1} H(u) d u$ est absolument convergente pour $\operatorname{Re} s>1$. On va voir qu'elle est alors égale à $\Gamma(s) F(s)$. Considérons un $s$ fixé, avec $\operatorname{Re} s=\sigma>1$. Soit $v>0$ fixé. Pour $u>0$,

$$
u^{s-1} H(u+v)=\sum_{n=1}^{\infty} G_{n}(u) \quad \text { où } \quad G_{n}(u)=a_{n} u^{s-1} e^{-n(u+v)} .
$$


On a

$$
\left|G_{n}(u)\right| \leq\left|a_{n}\right| e^{-n v} \cdot u^{\sigma-1} e^{-u}=G_{n}^{*}(u) .
$$

La série $\sum_{n=1}^{\infty} a_{n} e^{-n v}$ étant absolument convergente, $\sum_{n=1}^{\infty} G_{n}^{*}(u)$ est une fonction sommable sur l'intervalle $(0, \infty)$. On peut donc intégrer terme à terme sur cet intervalle la série $\sum_{n=1}^{\infty} G_{n}(u)$. On obtient ainsi

$$
\int_{0}^{\infty} u^{s-1} H(u+v) d u=\sum_{n=1}^{\infty} a_{n} \int_{0}^{\infty} u^{s-1} e^{-n(u+v)} d u
$$

ou, par le changement de variable $u=w / n$,

$$
\int_{0}^{\infty} u^{s-1} H(u+v) d u=\sum_{n=1}^{\infty} \frac{a_{n}}{n^{s}} \int_{0}^{\infty} w^{s-1} e^{-w-n v} d w=\Gamma(s) \sum_{n=1}^{\infty} \frac{a_{n}}{n^{s}} e^{-n v} .
$$

Cette relation vaut pour tout $v>0$.

Notons maintenant que, pour $u>0$,

$$
|H(u+v)| \leq M(u+v)^{-1} e^{-(u+v) / 2} \leq M u^{-1} e^{-u / 2}
$$

et par suite

$$
\left|u^{s-1} H(u+v)\right| \leq M u^{\sigma-2} e^{-u / 2} .
$$

Comme ceci est une fonction sommable sur l'intervalle $(0, \infty)$ et comme, quand $v$ tend vers zéro, $H(u+v)$ tend vers $H(u)$, on voit que, quand $v$ tend vers zéro,

$$
\int_{0}^{\infty} u^{s-1} H(u+v) d u \text { tend vers } \int_{0}^{\infty} u^{s-1} H(u) d u
$$

et par suite $\Gamma(s) \sum_{n=1}^{\infty}\left(a_{n} / n^{s}\right) e^{-n v}$ tend vers $\int_{0}^{\infty} u^{s-1} H(u) d u$. Mais, la série $\sum_{n=1}^{\infty} a_{n} / n^{s}$ étant convergente, $\sum_{n=1}^{\infty}\left(a_{n} / n^{s}\right) e^{-n v}$ tend vers $\sum_{n=1}^{\infty} a_{n} / n^{s}$, qui est égale à $F(s)$, d'après le théorème d'Abel d'après lequel, si la série $\sum_{n=0}^{\infty} u_{n}$ est convergente avec pour somme $S$, la somme de la série entière $\sum_{n=0}^{\infty} u_{n} x^{n}$, qui est convergente pour $|x|<1$, tend vers $S$ quand $x$ tend vers 1 par valeurs réelles $<1$.

On a donc, pour Re $s>1$,

$$
\int_{0}^{\infty} u^{s-1} H(u) d u=\Gamma(s) F(s)
$$

comme on l'a annoncé plus haut.

4.3. Maintenant, on peut écrire

$$
\int_{0}^{\infty} u^{s-1} H(u) d u=\int_{0}^{1} u^{s-1} H(u) d u+\int_{1}^{\infty} u^{s-1} H(u) d u,
$$


d'où, en faisant dans la première intégrale au second membre le changement de variable $u=1 / x$,

$$
\int_{0}^{\infty} u^{s-1} H(u) d u=\int_{1}^{\infty} x^{-s-1} H(1 / x) d x+\int_{1}^{\infty} u^{s-1} H(u) d u .
$$

Il résulte de l'inégalité (2) que l'intégrale $\int_{1}^{\infty} u^{s-1} H(u) d u$ est convergente pour tout $s$ et, quel que soit $\sigma_{0}$ réel, la convergence est uniforme pour $\operatorname{Re} s \leq$ $\sigma_{0}$. Sa valeur est donc une fonction entière de $s$.

Il en résulte que la fonction $F^{*}$ définie dans le domaine $D$ par

$$
F^{*}(s)=\Gamma(s) F(s)-\int_{1}^{\infty} u^{s-1} H(u) d u
$$

est méromorphe dans $D$ et a dans le demi-plan $\operatorname{Re} s \geq 1 / 2$ les mêmes pôles que $F$.

Il résulte de (3) et (4) que, pour $\operatorname{Re} s>1$,

$$
\int_{1}^{\infty} x^{-s-1} H(1 / x) d x=F^{*}(s) .
$$

4.4. On peut maintenant utiliser le lemme du paragraphe 3. Soit $\alpha$ l'abscisse de convergence de l'intégrale $\int_{1}^{\infty} x^{-s-1} H(1 / x) d x$. On a $\alpha \leq 1$ puisque l'intégrale converge pour $\operatorname{Re} s>1$.

D'autre part, $\alpha \geq \Theta$, où $\Theta$ est la borne supérieure des parties réelles des pôles de la fonction $F^{*}$, dont on sait qu'elle appartient à l'intervalle $[1 / 2,1]$.

En effet, l'intégrale est une fonction de $s$ holomorphe dans le demi-plan Re $s>\alpha$ et est égale à $F^{*}(s)$ pour $\operatorname{Re} s>1$. Or, quel que soit $\varepsilon>0, F^{*}$ a des pôles de partie réelle $>\Theta-\varepsilon$.

Le point $\alpha$ n'est pas un pôle de $F^{*}$ puisque cette fonction n'a pas de pôle sur le segment $[1 / 2,1]$.

Si $\alpha>1 / 2$, le lemme montre que, quel que soit $\varepsilon \in] 0, \alpha[$, quand $x$ tend vers $\infty$

$$
H(1 / x)=\Omega_{ \pm}\left(x^{\alpha-\varepsilon}\right) .
$$

En particulier $H(1 / x)=\Omega_{ \pm}\left(x^{1 / 2}\right)$.

Si $\alpha=1 / 2$, le lemme montre que

$$
H(1 / x)=\Omega_{ \pm}\left(x^{1 / 2}\right) .
$$

On a donc certainement

$$
H(1 / x)=\Omega_{ \pm}\left(x^{1 / 2}\right) \quad \text { quand } x \text { tend vers } \infty,
$$

ce qui équivaut à $H(u)=\Omega_{ \pm}\left(u^{-1 / 2}\right)$ quand $u$ tend vers zéro. Le théorème est ainsi démontré. 
5. Nous allons maintenant donner des exemples simples de suites satisfaisant aux hypothèses du théorème. Dans chacun de ces exemples, le fait que le rayon de convergence de la série $\sum_{n=1}^{\infty} a_{n} x^{n}$ est égal à 1 est évident. Nous n'aurons à vérifier que les propriétés 2) et 3 ).

5.1. Tout d'abord, les hypothèses sont évidemment satisfaites par $a_{n}=$ $\mu(n)$. Donc, quand $x$ tend vers 1 , d'une part

$$
\sum_{n=1}^{\infty} \mu(n) x^{n}=o\left(\frac{1}{1-x}\right),
$$

d'autre part

$$
\sum_{n=1}^{\infty} \mu(n) x^{n}=\Omega_{ \pm}\left(\frac{1}{\sqrt{1-x}}\right) .
$$

Cette dernière relation entraîne que, quand $x$ tend vers $\infty$,

$$
M(x)=\Omega_{ \pm}\left(x^{1 / 2}\right) .
$$

Ceci est d'ailleurs un résultat connu (cf. par exemple, [1], page 99).

5.2. Les hypothèses du théorème sont aussi satisfaites par la suite $a_{n}=$ $(-1)^{n-1} \mu(n)$. On vérifie aisément, soit directement, soit en remarquant que $n \mapsto(-)^{n-1} \mu(n)$ est une fonction multiplicative et considérant ses valeurs pour les puissances des nombres premiers, que cette fonction est la convolution $\mu * f$ où $f$ est la fonction (multiplicative) définie par $f(1)=1$ et, pour $n>1$,

$$
f(n)= \begin{cases}2 & \text { si } n \text { est une puissance de } 2, \\ 0 & \text { dans le cas contraire }\end{cases}
$$

La série de Dirichlet $\sum_{n=1}^{\infty}(-1)^{n-1} \mu(n) / n^{s}$ est donc le produit de Dirichlet des séries $\sum_{n=1}^{\infty} \mu(n) / n^{s}$ et $\sum_{n=1}^{\infty} f(n) / n^{s}$.

Pour $s=1$, la première est convergente et la seconde absolument convergente. La série $\sum_{n=1}^{\infty}(-1)^{n-1} \mu(n) / n$ est donc convergente.

Pour $\operatorname{Re} s>1$, on a

$$
\sum_{n=1}^{\infty} \frac{(-1)^{n-1} \mu(n)}{n^{s}}=\frac{1}{\zeta(s)}\left(1+\sum_{k=1}^{\infty} \frac{2}{2^{k s}}\right)=\frac{1}{\zeta(s)} \cdot \frac{2^{s}+1}{2^{s}-1} .
$$

Ceci est une fonction méromorphe dans $\mathbb{C}$, ayant comme pôles dans le demiplan $\operatorname{Re} s \geq 1 / 2$ les zéros de la fonction $\zeta$.

Le théorème montre que, quand $x$ tend vers 1 , alors que

on a

$$
\sum_{n=1}^{\infty}(-1)^{n-1} \mu(n) x^{n}=o\left(\frac{1}{1-x}\right)
$$

$$
\sum_{n=1}^{\infty}(-1)^{n-1} \mu(n) x^{n}=\Omega_{ \pm}\left(\frac{1}{\sqrt{1-x}}\right) .
$$


En remarquant que

$$
\sum_{n=1}^{\infty} \mu(n) x^{n}=-\sum_{n=1}^{\infty}(-1)^{n-1} \mu(n)(-x)^{n}
$$

on voit que, quand $x$ tend vers -1 ,

$$
\sum_{n=1}^{\infty} \mu(n) x^{n}=o\left(\frac{1}{1+x}\right) \quad \text { et } \quad \sum_{n=1}^{\infty} \mu(n) x^{n}=\Omega_{ \pm}\left(\frac{1}{\sqrt{1+x}}\right) .
$$

5.3. Les hypothèses du théorème sont encore satisfaites si $a_{n}=\lambda(n)-$ $q(n) / \zeta(1 / 2)$, où $\lambda$ est la fonction de Louville et

$$
q(n)= \begin{cases}1 & \text { si } n \text { est un carré } \\ 0 & \text { dans le cas contraire. }\end{cases}
$$

On sait que, pour $\operatorname{Re} s>1$,

$$
\sum_{n=1}^{\infty} \frac{\lambda(n)}{n^{s}}=\frac{\zeta(2 s)}{\zeta(s)}=\left(\sum_{n=1}^{\infty} \frac{\mu(n)}{n^{s}}\right)\left(\sum_{n=1}^{\infty} \frac{q(n)}{n^{s}}\right) .
$$

Il s'ensuit que la série $\sum_{n=1}^{\infty} \lambda(n) / n^{s}$ est le produit de Dirichlet des séries $\sum_{n=1}^{\infty} \mu(n) / n^{s}$ et $\sum_{n=1}^{\infty} q(n) / n^{s}$.

Pour $s=1$, la première est convergente et la seconde absolument convergente. La série $\sum_{n=1}^{\infty} \lambda(n) / n$ est donc convergente (avec pour somme 0 ). On voit ainsi que la série $\sum_{n=1}^{\infty} a_{n} / n$ est convergente.

Pour $\operatorname{Re} s>1$,

$$
\begin{aligned}
\sum_{n=1}^{\infty} \frac{a_{n}}{n^{s}} & =\sum_{n=1}^{\infty} \frac{\lambda(n)}{n^{s}}-\frac{1}{\zeta(1 / 2)} \sum_{n=1}^{\infty} \frac{q(n)}{n^{s}}=\frac{\zeta(2 s)}{\zeta(s)}-\frac{1}{\zeta(1 / 2)} \zeta(2 s) \\
& =\zeta(2 s)\left(\frac{1}{\zeta(s)}-\frac{1}{\zeta(1 / 2)}\right) .
\end{aligned}
$$

Ceci est une fonction méromorphe dans $\mathbb{C}$ ayant comme pôles dans le demiplan $\operatorname{Re} s \geq 1 / 2$ les zéros de la fonction $\zeta$.

Le théorème montre que, quand $x$ tend vers 1 ,

$$
\sum_{n=1}^{\infty}\left(\lambda(n)-\frac{q(n)}{\zeta(1 / 2)}\right) x^{n}=o\left(\frac{1}{1-x}\right)
$$

et

$$
\sum_{n=1}^{\infty}\left(\lambda(n)-\frac{q(n)}{\zeta(1 / 2)}\right) x^{n}=\Omega_{ \pm}\left(\frac{1}{\sqrt{1-x}}\right),
$$

ce qui entraîne que, quand $x$ tend vers $\infty$,

$$
\sum_{n \leq x}\left(\lambda(n)-\frac{q(n)}{\zeta(1 / 2)}\right)=\Omega_{ \pm}\left(x^{1 / 2}\right) .
$$


Ces relations peuvent se traduire par

$$
\sum_{n=1}^{\infty} \lambda(n) x^{n}-\frac{\sqrt{\pi}}{2 \zeta(1 / 2)} \cdot \frac{1}{\sqrt{1-x}}=o\left(\frac{1}{1-x}\right)
$$

et

$$
\sum_{n=1}^{\infty} \lambda(n) x^{n}-\frac{\sqrt{\pi}}{2 \zeta(1 / 2)} \cdot \frac{1}{\sqrt{1-x}}=\Omega_{ \pm}\left(\frac{1}{\sqrt{1-x}}\right) \quad \text { quand } x \rightarrow 1,
$$

et

$$
\sum_{n \leq x} \lambda(n)-\frac{x^{1 / 2}}{\zeta(1 / 2)}=\Omega_{ \pm}\left(x^{1 / 2}\right) \quad \text { quand } x \rightarrow \infty .
$$

Cette dernière relation est proposée comme exercice dans [1], page 102.

5.4. On peut obtenir des informations sur le comportement de la série $\sum_{n=1}^{\infty} \lambda(n) x^{n}$ quand $x$ tend vers -1 en considérant

$$
a_{n}=(-1)^{n-1} \lambda(n)-\frac{1+\sqrt{2}}{\zeta(1 / 2)} q(n) .
$$

Etant donné $X>1$, on a, pour tout $s$,

$$
\begin{aligned}
\sum_{n \leq X} \frac{(-1)^{n-1} \lambda(n)}{n^{s}} & =\sum_{\substack{n \leq X \\
n \text { impair }}} \frac{\lambda(n)}{n^{s}}-\sum_{\substack{n \leq X \\
n \text { pair }}} \frac{\lambda(n)}{n^{s}} \\
& =\sum_{n \leq X} \frac{\lambda(n)}{n^{s}}-2 \sum_{\substack{n \leq X \\
n \text { pair }}} \frac{\lambda(n)}{n^{s}} \\
& =\sum_{n \leq X} \frac{\lambda(n)}{n^{s}}-2 \sum_{m \leq X / 2} \frac{\lambda(2 m)}{(2 m)^{s}} \\
& =\sum_{n \leq X} \frac{\lambda(n)}{n^{s}}+2^{1-s} \sum_{m \leq X / 2} \frac{\lambda(m)}{m^{s}} .
\end{aligned}
$$

On voit ainsi que la série $\sum_{n=1}^{\infty}(-1)^{n-1} \lambda(n) / n$ est convergente (avec pour somme 0 ), de sorte que la série $\sum_{n=1}^{\infty} a_{n} / n$ est convergente, et que, pour $\operatorname{Re} s>1$,

$$
\sum_{n=1}^{\infty} \frac{a_{n}}{n^{s}}=\left(1+2^{1-s}\right) \frac{\zeta(2 s)}{\zeta(s)}-\frac{1+\sqrt{2}}{\zeta(1 / 2)} \zeta(2 s)=\zeta(2 s)\left(\frac{1+2^{1-s}}{\zeta(s)}-\frac{1+\sqrt{2}}{\zeta(1 / 2)}\right) .
$$

Ceci est encore une fonction méromorphe dans $\mathbb{C}$ ayant comme pôles dans le demi-plan $\operatorname{Re} s \geq 1 / 2$ les zéros de la fonction $\zeta$. 
Le théorème montre que, quand $x$ tend vers 1 ,

$$
\begin{aligned}
\sum_{n=1}^{\infty}\left((-1)^{n-1} \lambda(n)-\frac{1+\sqrt{2}}{\zeta(1 / 2)} q(n)\right) x^{n} & =o\left(\frac{1}{1-x}\right) \\
& =\Omega_{ \pm}\left(\frac{1}{\sqrt{1+x}}\right)
\end{aligned}
$$

ce qui donne

$$
\begin{aligned}
\sum_{n=1}^{\infty}(-1)^{n-1} \lambda(n) x^{n}-\frac{(1+\sqrt{2}) \sqrt{\pi}}{2 \zeta(1 / 2)} \cdot \frac{1}{\sqrt{1-x}} & =o\left(\frac{1}{1-x}\right) \\
& =\Omega_{ \pm}\left(\frac{1}{\sqrt{1-x}}\right)
\end{aligned}
$$

Il en résulte que, quand $x$ tend vers -1 ,

$$
\begin{aligned}
\sum_{n=1}^{\infty} \lambda(n) x^{n}-\frac{(1+\sqrt{2}) \sqrt{\pi}}{2 \zeta(1 / 2)} \cdot \frac{1}{\sqrt{1+x}} & =o\left(\frac{1}{1+x}\right) \\
& =\Omega_{ \pm}\left(\frac{1}{\sqrt{1+x}}\right)
\end{aligned}
$$

5.5. Les hypothèses du théorème sont satisfaites si $a_{n}=(-1)^{\omega(n)}$, où $\omega(n)$ est le nombre des nombres premiers qui divisent $n$. Tout d'abord, on voit facilement que la fonction $n \rightarrow(-1)^{\omega(n)}$ est la convolution $\mu * f$ où $f$ est la fonction multiplicative déterminée par

$$
f\left(p^{k}\right)=1-k \quad \text { pour tout } p \text { premier et tout } k \in \mathbb{N}^{*} .
$$

La série $\sum_{n=1}^{\infty}(-1)^{\omega(n)} / n^{s}$ est donc le produit de Dirichlet de $\sum_{n=1}^{\infty} \mu(n) / n^{s}$ et $\sum_{n=1}^{\infty} f(n) / n^{s}$.

Pour $s=1$, la première est convergente et la seconde est absolument convergente, car, pour $\operatorname{Re} s=\sigma>0$,

$$
\sum_{k=1}^{\infty}\left|\frac{f\left(p^{k}\right)}{p^{k s}}\right|=\frac{1}{\left(p^{\sigma}-1\right)^{2}} .
$$

La série $\sum_{n=1}^{\infty}(-1)^{\omega(n)} / n$ est donc convergente (avec pour somme 0 ).

Pour $\operatorname{Re} s>1$, les deux séries sont absolument convergentes et on a

$$
\sum_{n=1}^{\infty} \frac{(-1)^{\omega(n)}}{n^{s}}=\left(\sum_{n=1}^{\infty} \frac{\mu(n)}{n^{s}}\right) \sum_{n=1}^{\infty} \frac{f(n)}{n^{s}}=\frac{1}{\zeta(s)} \sum_{n=1}^{\infty} \frac{f(n)}{n^{s}} .
$$

Comme

$$
\sum_{k=1}^{\infty} \frac{f\left(p^{k}\right)}{p^{k s}}=-\frac{1}{\left(p^{s}-1\right)^{2}}
$$


on a

$$
\sum_{n=1}^{\infty} \frac{f(n)}{n^{s}}=\prod\left(1-\frac{1}{\left(p^{s}-1\right)^{2}}\right)
$$

On vérifie facilement que

$$
1-\frac{1}{\left(p^{s}-1\right)^{2}}=\left(1-\frac{1}{p^{2 s}}\right)\left(1-\frac{2 p^{s}-1}{\left(p^{s}+1\right)\left(p^{s}-1\right)^{3}}\right) .
$$

Pour $\operatorname{Re} s>1$, les produits

$$
\prod\left(1-\frac{1}{p^{2 s}}\right) \text { et } \prod\left(1-\frac{2 p^{s}-1}{\left(p^{s}+1\right)\left(p^{s}-1\right)^{3}}\right)
$$

sont absolument convergents et on a donc

$$
\begin{aligned}
\sum_{n=1}^{\infty} \frac{f(n)}{n^{s}} & =\left(\prod\left(1-\frac{1}{p^{2 s}}\right)\right) \prod\left(1-\frac{2 p^{s}-1}{\left(p^{s}+1\right)\left(p^{s}-1\right)^{3}}\right) \\
& =\frac{1}{\zeta(2 s)} \prod\left(1-\frac{2 p^{s}-1}{\left(p^{s}+1\right)\left(p^{s}-1\right)^{3}}\right)
\end{aligned}
$$

et par suite

$$
\sum_{n=1}^{\infty} \frac{(-1)^{\omega(n)}}{n^{s}}=\frac{1}{\zeta(s) \zeta(2 s)} \prod\left(1-\frac{2 p^{s}-1}{\left(p^{s}+1\right)\left(p^{s}-1\right)^{3}}\right) .
$$

Notons maintenant que, pour $\operatorname{Re} s=\sigma>0$,

$$
\left|\frac{2 p^{s}-1}{\left(p^{s}+1\right)\left(p^{s}-1\right)^{3}}\right| \leq \frac{2 p^{\sigma}+1}{\left(p^{\sigma}-1\right)^{4}}
$$

et par suite, quel que soit $\sigma_{0}>1 / 3$, le produit dans (5) est uniformément convergent pour $\operatorname{Re} s \geq \sigma_{0}$. Soit $\Phi(s)$ sa valeur. $\Phi$ est une fonction holomorphe dans le demi-plan $\operatorname{Re} s>1 / 3$ et on voit facilement que $\Phi(s) \neq 0$ quand $\operatorname{Re} s=1 / 2$. D'après (5), pour $\operatorname{Re} s>1$,

$$
\sum_{n=1}^{\infty} \frac{(-1)^{\omega(n)}}{n^{s}}=\frac{\Phi(s)}{\zeta(s) \zeta(2 s)}
$$

Ceci est une fonction méromorphe dans le demi-plan $\operatorname{Re} s>1 / 3$, dont les pôles dans le demi-plan $\operatorname{Re} s \geq 1 / 2$ sont des zéros de la fonction $\zeta$ et qui a en particulier pour pôles les zéros de $\zeta$ de partie réelle $1 / 2$.

Le théorème montre que, quand $x$ tend vers 1 ,

$$
\begin{aligned}
\sum_{n=1}^{\infty}(-1)^{\omega(n)} x^{n} & =o\left(\frac{1}{1-x}\right) \\
& =\Omega_{ \pm}\left(\frac{1}{\sqrt{1-x}}\right)
\end{aligned}
$$


ce qui entraîne que

$$
\sum_{n \leq x}(-1)^{\omega(n)}=\Omega_{ \pm}\left(x^{1 / 2}\right) \quad \text { quand } x \text { tend vers l'infini. }
$$

5.6. Comme dernier exemple considérons $a_{n}=\Lambda(n)-1$, où $\Lambda$ est la fonction de von Mangoldt. Il résulte immédiatement du théorème des nombres premiers sous la forme " $\psi(x) \sim x$ quand $x$ tend vers $\infty$ " que, quand $x$ tend vers $\infty$,

$$
\sum_{n \leq x} a_{n}=o(x)
$$

Pour Re $s>1$,

$$
\sum_{n=1}^{\infty} \frac{a_{n}}{n^{s}}=\sum_{n=1}^{\infty} \frac{\Lambda(n)}{n^{s}}-\sum_{n=1}^{\infty} \frac{1}{n^{s}}=-\frac{\zeta^{\prime}(s)}{\zeta(s)}-\zeta(s) .
$$

Ceci est une fonction méromorphe dans $\mathbb{C}$, ayant comme pôles les zéros de $\zeta$.

Le théorème montre que, quand $x$ tend vers 1 ,

$$
\begin{aligned}
\sum_{n=1}^{\infty}(\Lambda(n)-1) x^{n} & =o\left(\frac{1}{1-x}\right) \\
& =\Omega_{ \pm}\left(\frac{1}{\sqrt{1-x}}\right)
\end{aligned}
$$

ce qui entraîne que, quand $x$ tend vers $\infty$,

$$
\psi(x)-x=\Omega_{ \pm}\left(x^{1 / 2}\right) .
$$

En fait, il est connu que

$$
\psi(x)-x=\Omega_{ \pm}\left(x^{1 / 2} \log \log \log x\right),
$$

mais c'est beaucoup plus difficile à démontrer (cf. [2], page 100).

Les deux premières relations donnaient

$$
\begin{aligned}
\sum_{n=1}^{\infty} \Lambda(n) x^{n}-\frac{1}{1-x} & =o\left(\frac{1}{1-x}\right) \\
& =\Omega_{ \pm}\left(\frac{1}{\sqrt{1-x}}\right)
\end{aligned}
$$

quand $x$ tend vers 1 .

\section{Références}

[1] A. Blanchard, Initiation à la Théorie Analytique des Nombres Premiers, Dunod, 1969.

[2] A. E. Ingham, The Distribution of Prime Numbers, Cambridge Tracts in Math. and Math. Phys. 30, Cambridge Univ. Press, 1932. 
[3] E. Landau, Über einen Satz von Tschebyschef, Math. Ann. 61 (1905), 527-550.

Université de Paris-Sud

Mathématique, bt. 425

91405 Orsay, France

Reçu le 25.8.1998

et révisé le 9.3.1999 